\title{
Dietary $n-6$ and $n-3$ PUFA alter the free oxylipin profile differently in male and female rat hearts
}

\author{
Afroza Ferdouse, Shan Leng, Tanja Winter and Harold M. Aukema* \\ Department of Food and Human Nutritional Sciences, University of Manitoba, Winnipeg, Canada; Canadian Centre for Agri- \\ Food Research in Health and Medicine, Winnipeg, Canada \\ (Submitted 3 November 2018 - Final revision received 28 March 2019 - Accepted 8 May 2019)
}

\begin{abstract}
Oxylipins are bioactive lipid mediators synthesised from PUFA. The most well-known oxylipins are the eicosanoids derived from arachidonic acid (ARA), and many of them influence cardiac physiology in health and disease. Oxylipins are also formed from other $n$ - 3 and $n-6$ PUFA such as $\alpha$-linolenic acid (ALA), EPA, DHA and linoleic acid (LA), but fundamental data on the heart oxylipin profile, and the effect of diet and sex on this profile, are lacking. Therefore, weanling female and male Sprague-Dawley rats were given American Institute of Nutrition (AIN)-93G-based diets modified in oil composition to provide higher levels of ALA, EPA, DHA, LA and LA + ALA, compared with control diets. After 6 weeks, free oxylipins in rat hearts were increased primarily by their precursor PUFA, except for EPA oxylipins, which were increased not only by dietary EPA but also by dietary ALA or DHA. Dietary DHA had a greater effect than ALA or EPA on reducing ARA oxylipins. An exception to the dietary $n$-3 PUFA-lowering effects on ARA oxylipins was observed for several ARA-derived PG metabolites that were higher in rats given EPA diets. Higher dietary LA increased LA oxylipins, but it had no effect on ARA oxylipins. Overall, heart oxylipins were higher in female rats, but this depended on dietary treatment: the female oxylipin:male oxylipin ratio was higher in rats provided the ALA compared with the DHA diet, with other diet groups having ratios in between. In conclusion, individual PUFA and sex have unique and interactive effects on the rat heart free oxylipin profile.
\end{abstract}

Key words: Oxylipins: Eicosanoids: Heart: Sex: Dietary fat: $\alpha$-Linolenic acid: EPA: DHA: Linoleic acid: Arachidonic acid

There is substantial literature on fatty acid compositions in mammalian heart, but little is known about their oxygenated bioactive metabolites called oxylipins. The most well-studied class of oxylipins are the eicosanoids generated by cyclo-oxygenase, lipoxygenase and cytochrome $\mathrm{P} 450$ oxygenase activities from arachidonic acid (ARA, $20: 4 n-6$ ); these bioactive lipids are the major mediators of ARA effects in the body ${ }^{(1,2)}$. Oxylipins also are synthesised from other PUFA such as EPA, DHA, $\alpha$-linolenic acid (ALA, $18: 3 n-3$ ) and linoleic acid (LA, $18: 2 n-6$ ). Oxylipins have many biological functions, and several effects of free oxylipins on the heart in health and disease have been shown ${ }^{(3,4)}$. For example, ARA-derived $\mathrm{PGE}_{2}$ modulates cardiac contractility depending on which receptor it activates $^{(5)}$, 19-hydroxyeicosatetraenoic acid (19-HETE) reduces cardiomyocyte hypertrophy ${ }^{(6)}$, 20-HETE and epoxy-eicosatrienoic acids (EpETrE) induce cardiac hypertrophy ${ }^{(7)}$, EpETrE prevent lipopolysaccharide-induced cardiac dysfunction ${ }^{(8)}$ and 5-, 12and 15-HETE induce cellular hypertrophy in human ventricular cardiomyocytes $^{(9)}$. Oxylipins derived from other PUFA also have effects on the heart, such as EPA-derived 18-hydroxy- eicosapentaenoic acid (18-HEPE), which is anti-inflammatory in cardiac fibroblasts ${ }^{(10)}$, and DHA-derived 13,14-epoxydocosapentaenoic acid (13,14-EpDPE), which potently dilates porcine coronary microvessels ${ }^{(11)}$. Little is known about the existence and functions of heart oxylipins derived directly from 18-carbon PUFA (LA and ALA), although there is some evidence that LA affects heart function indirectly by altering ARA-derived oxylipins ${ }^{(12)}$. LA-derived oxylipins such as 13hydroxy-octadecadienoic acid have been shown to attenuate platelet adhesion to endothelial cells ${ }^{(13)}$, and dihydroxyoctadecenoic acids may induce oxidative stress and proinflammatory events in vascular endothelial cells ${ }^{(14)}$, but direct effects of LA oxylipins on the heart have not been reported. Furthermore, there is little information on the heart oxylipin profile in the literature ${ }^{(15)}$. Thus, the first objective of the present study was to provide a comprehensive profile of the free oxylipins in the rat heart.

Dietary PUFA effects on oxylipins in different tissues have historically been predicted based on their effects on tissue PUFA composition. However, comparisons of PUFA and

Abbreviations: ALA, $\alpha$-linolenic acid; ARA, arachidonic acid; DGLA, dihomo- $\boldsymbol{\gamma}$-linolenic acid; EpDPE, epoxy-docosapentaenoic acid; EpETrE, epoxy-eicosatrienoic acid; HEPE, hydroxy-eicosapentaenoic acid; HETE, hydroxy-eicosatetraenoic acid; LA, linoleic acid; NL, neutral lipid; PL, phospholipid. 
oxylipin profiles in rat kidney, liver and adipose reveal that oxylipins do not always reflect their parent PUFA levels. Further, tissue oxylipin levels are not only modulated by dietary alterations in their direct fatty acid precursors ${ }^{(16-21)}$ but also can be altered by dietary fatty acids that are not their direct precursors, such as ARA oxylipins being reduced in the heart by dietary $n-3$ fatty acids $^{(22)}$, or being increased by dietary $\mathrm{LA}^{(12)}$. Thus, the second objective of the present study was to examine the effect of differing dietary oils on the heart oxylipin profile.

Oxylipin profiling in the heart also may be relevant to further understanding of sex differences in the normal and diseased heart ${ }^{(4)}$. Previous oxylipin profiling studies demonstrated higher levels of renal, hepatic and serum oxylipins in male rats ${ }^{(23,24)}$. In several adipose depots, sex differences depended on the diet: for example, rats provided a diet rich in DHA had higher adipose oxylipins in males, while those provided diets enriched in either ALA or EPA had higher oxylipins in females ${ }^{(21)}$. There are few data on sex differences in heart oxylipins, but some studies indicate that some individual ARA-derived oxylipins may be higher in females in vascular tissue ${ }^{(25,26)}$. Therefore, the third objective of the present study was to examine sex differences in the heart profile.

To achieve these three objectives, female and male rats were provided diets that differed only in oil composition so that each test diet compared with control had higher levels of specific PUFA. Rats were used since collection of hearts from humans consuming specific diets is not possible, and rats are a good model of human lipid metabolism for a whole body approach.

\section{Materials and methods}

\section{Animals and diets}

A total number of seventy-two female and male SpragueDawley weanling rats were randomly provided six different diets for 6 weeks as described in detail in references ${ }^{(21,23,24)}$, which describe diet and sex effects on kidney, liver, serum and adipose oxylipins in these rats. Briefly, the diets were based on the standard American Institute of Nutrition (AIN)-93G diet but had $10 \mathrm{~g}$ oil instead of $7 \mathrm{~g}$ oil per $100 \mathrm{~g}$ diet. Oil blends were designed for each diet so that the control diet had adequate levels of the essential fatty acids, ALA and LA, and high levels of MUFA. In the test diets, MUFA were replaced with oils high in ALA, EPA, DHA and LA so that these diets contained $3 \mathrm{~g}$ (per $100 \mathrm{~g}$ diet) more of each of these PUFA, respectively, compared with the control diet. The LA + ALA diet also contained 3 g more LA per $100 \mathrm{~g}$ diet and had additional ALA so that the LA:ALA ratio was similar to the control diet. The percentage of energy from LA and ALA in all diets except the ALA diet is within the range of human consumption patterns. The higher amounts of ALA, EPA and DHA in the ALA, EPA and DHA diets, respectively, compared with control, were matched with the increased amount of LA in the LA compared with control diet, so that direct comparisons could be made between PUFA. However, the levels of these $n$ - 3 PUFA in the ALA, EPA and DHA diets are not commonly achieved in the human population. The levels of unsaturated fatty acids and SFA were similar across all diets.
The rats were housed individually, weighed weekly and the dietary intervention was carried out for all rats during the same 6-week period. Rats were anaesthetised using isofluorane before termination in random order and harvested heart tissues were weighed, immediately frozen in liquid $\mathrm{N}_{2}$ and stored at $-80^{\circ} \mathrm{C}$ until analysis. All animal procedures were performed according to the Canadian Council for Animal Care guidelines and approved by the University of Manitoba Animal Care Committee.

\section{Free oxylipin analysis}

Hearts were rinsed with ice-cold Tyrode's salt ( $\mathrm{pH}$ 7.6) solution to remove blood before homogenisation of the whole heart in fresh Tyrode's solution. An optimum amount of heart homogenate ( $400 \mu$ l containing $70 \mathrm{mg}$ tissue) for oxylipin quantification was determined from a dose-response curve, and analysis of oxylipins was performed by HPLC/MS/MS as described ${ }^{(20,23)}$. Briefly, deuterated internal standards (Cayman Chemicals) were added to sample homogenates containing antioxidants and adjusted to $\mathrm{pH}<3 \cdot 0$. Strata-X-SPE (Phenomenex) columns preconditioned with methanol followed by $\mathrm{pH} 3.0$ water were used for solid phase extraction. After column loading and washing, free oxylipins were eluted with $100 \%$ methanol. Samples obtained were dried and re-suspended in the mobile phase (water/acetonitrile/formic acid, 70/30/0.02, v/v/v) for oxylipin analysis by HPLC/MS/MS (QTRAP 6500; Sciex). Quantification of oxylipins was performed using the stable isotope dilution $\operatorname{method}^{(27)}$. Oxylipins screened but below the level of detection ( $<3$ times above baseline) and those detected but below the level of quantification ( $>3$ to $<5$ times above baseline) are provided in online Supplementary Table S1a and $1 \mathrm{~b}$, respectively. Further details of all oxylipins scanned, mass transitions, internal standards, standard curve slopes and retention times are provided in references ${ }^{(20,23)}$.

\section{Fatty acid analysis}

Total lipids were extracted from $250 \mu$ of the heart homogenate and fatty acids were analysed as described ${ }^{(19,28)}$. Briefly, after solvent extraction of total lipids, total phospholipid (PL) and neutral lipid (NL) were separated by TLC (heptane-isopropyl ether-acetic acid, 60:40:3, by vol. $)^{(29)}$. Fatty acids were methylated using methanolic $\mathrm{H}_{2} \mathrm{SO}_{4}$, extracted in hexane and analysed by GC as described ${ }^{(30,31)}$. Analyses of both fatty acids and oxylipins were performed in random order.

\section{Statistical analysis}

With six rats in each diet/sex group (total of seventy-two rats), this study had a power of 0.8 to detect an effect size of 0.45 at a significance level of $P<0.05$ (G*Power Software version 3.1.9.2). Statistical analysis was performed using SAS Software Version 9.3 (SAS Institute Inc). The Shapiro-Wilk test was performed to test for normality, followed by oneor two-way ANOVA if data were distributed normally. The Kruskal-Wallis test was performed when data were not normal even after transformation. Tukey's test was used for post hoc analyses. Observations greater than the mean $\pm 3 \mathrm{SD}$ of 
a group were removed as outliers. All data are reported as means with their standard errors.

\section{Results \\ Overall oxylipin and fatty acid distributions}

Out of 164 oxylipins scanned, seventy-five were detected and quantified in the rat hearts. Approximately two-thirds of the oxylipins were derived from $n-6$ PUFA; two-thirds of these were formed from ARA. Approximately half of the remaining $n-3$ PUFA-derived oxylipins were formed from DHA, one-third were from EPA and one-fifth were from ALA (online Supplementary Tables S2 and S3).

The proportions of oxylipin mass did not necessarily reflect PUFA mass proportions in either the PL or NL fractions. For example, in the control group, the proportion of LA and ARA oxylipins was approximately two-thirds and one-third, respectively, while the proportion of LA and ARA was almost equally split between these two PUFA in the PL fraction and skewed more towards LA in the NL fraction (Fig. 1(A)). Further, the distributions of oxylipin and PUFA mass also were different with the different dietary oil treatments; examples of the DHA and LA groups are shown in Fig. 1(B) and (C), respectively; values for all diets can be found in online Supplementary Table S4.

\section{Effects of dietary PUFA on n-3-derived oxylipins}

At the end of the feeding period, there were no differences in body or heart weights between the dietary treatments. Heart and body weights were higher in males, while heart weights relative to body weight were higher in females (online Supplementary Table S5). Compared with the control group, ALA and DHA oxylipins were higher only in hearts from rats given diets that were higher in their specific precursor PUFA (Fig. 2(A); online Supplementary Table S2). On the other hand, EPA oxylipins were higher not only in EPA rats but also in rats given ALA and DHA diets. ALA and DHA diets did not increase EPA oxylipins as much as with the EPA diet, however. The LA + ALA diet with higher levels of ALA than the control diet, but lower levels than in the ALA diet, also increased ALA and EPA oxylipins when compared with the control diet, but fewer oxylipins were affected and the effect was smaller than in hearts from rats given the ALA diet. The LA diet did not affect the $n$-3 oxylipin levels in the rat heart, except for 13-hydroxyoctadecatrienoic acid.

To compare the levels of oxylipins with their PUFA precursor, the fatty acid compositions of the PL and NL fractions were analysed (Fig. 2(C); online Supplementary Tables S6 and S7). Similar to oxylipin effects, $n$-3 PUFA in heart PL were increased in rats provided their specific precursor PUFA (Fig. 2(C); online Supplementary Table S6). However, effects on other $n-3$ PUFA did not necessarily reflect oxylipin changes. For example, dietary EPA significantly reduced DHA in the PL fraction, even though all DHA oxylipins (except 13-hydroxy-DHA) were not different in rats given this diet. The results of the NL PUFA analyses were similar to the PL results - that is, $n-3$ PUFA in heart NL also were generally higher in rats provided their specific precursor PUFA, but other $n$-3 PUFA were not significantly altered, even if their oxylipins were higher (Fig. 2(C); online Supplementary Table S7).

\section{Effects of dietary PUFA on n-6-derived oxylipins}

Compared with the control group, the LA diet increased six out of ten LA-derived oxylipins, with the remaining LA oxylipins following the same trend (Fig. 2(B); online Supplementary Table S3). The LA diet also increased the one oxylipin derived from $\gamma$-linolenic acid, but very few other $n-6$ oxylipins were altered by dietary LA; only one of three dihomo- $\gamma$-linolenic acid (DGLA) and one of thirty ARA oxylipins were higher in female hearts. The LA + ALA diet had a similar level of LA as the LA diet, but also had a higher level of ALA compared with the control diet to achieve the same LA:ALA ratio as the control diet. $n$ - 6 Oxylipin levels in rats given this diet were similar to those given the LA diet, although there were fewer differences between the LA + ALA and the control diet than was the case for the LA compared with control diet.

Higher levels of dietary $n$-3 PUFA, however, resulted in lower levels of ARA-derived oxylipins, with little effect on oxylipins derived from other n-6 PUFA (LA, DGLA) (Fig. 2(B); Supplementary Table S3). The effect of individual dietary $n$-3 PUFA on ARA oxylipins differed, with DHA having the greatest effect: twenty-five out of thirty ARA oxylipins quantified were lower in hearts from DHA compared with control rats, while only nine and ten were lower in hearts from rats given the ALA and EPA diets, respectively.

As was the case with the $n$ - 3 PUFA, the changes in $n-6$ PUFA in the PL and NL sometimes reflected $n-6$ oxylipin changes (e.g. ARA in the PL fraction in hearts from rats given EPA and DHA diets), but other times did not (e.g. ARA in the PL and NL fractions in hearts from rats given ALA diets followed the same lowering trend but were not significantly different) (Fig. 2(A) and (C)). Another example of how oxylipin levels can differ from their precursor PUFA levels is illustrated by the higher levels of some ARA-derived PG metabolites (i.e. 15-deoxy- $\mathrm{PGD}_{2}$ and 15-keto-PGE $\mathrm{P}_{2}$ ) in hearts from rats given the EPA diet, while twelve other ARA oxylipins were lower (Fig. 2(B)).

\section{Sex effects in oxylipin and PUFA levels}

Previous studies in these rats showed that males generally had higher levels of oxylipins in kidney and liver, except for the DHA-derived oxylipins in kidney, which were generally higher in females ${ }^{(23)}$. Hence sex differences in oxylipins were examined in the heart. These analyses revealed that all twenty-seven oxylipins with a main sex effect were higher in females, and that twenty-one of the twenty-two with a sex by diet interaction had higher oxylipins in females in at least one group. Only thromboxane $\mathrm{B}_{2}$ was higher in males given the LA + ALA diet (online Supplementary Table S3). However, sex differences were often not consistent across all groups, even within significant main effects. To further examine these interaction effects, the female oxylipin:male oxylipin mass ratio was calculated for each oxylipin, and the effect of diet on this ratio was tested. This analysis showed an interaction between diet and oxylipins 
(A)

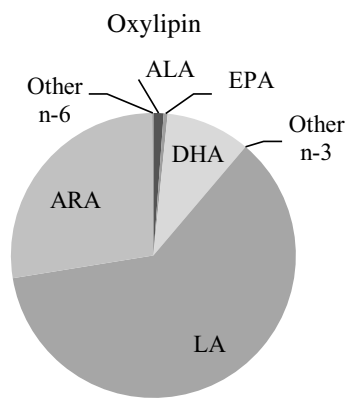

(B)

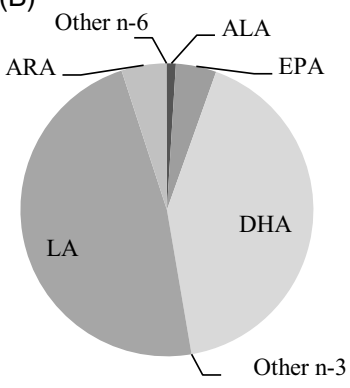

(C)

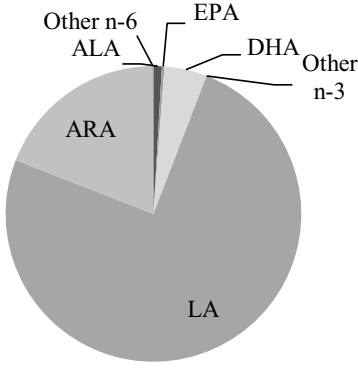

PUFA-PL
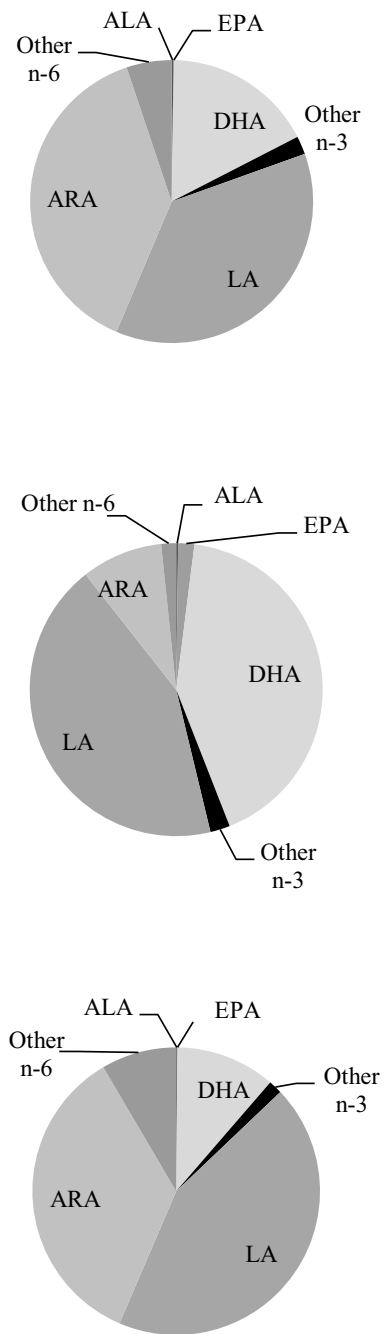

PUFA-NL
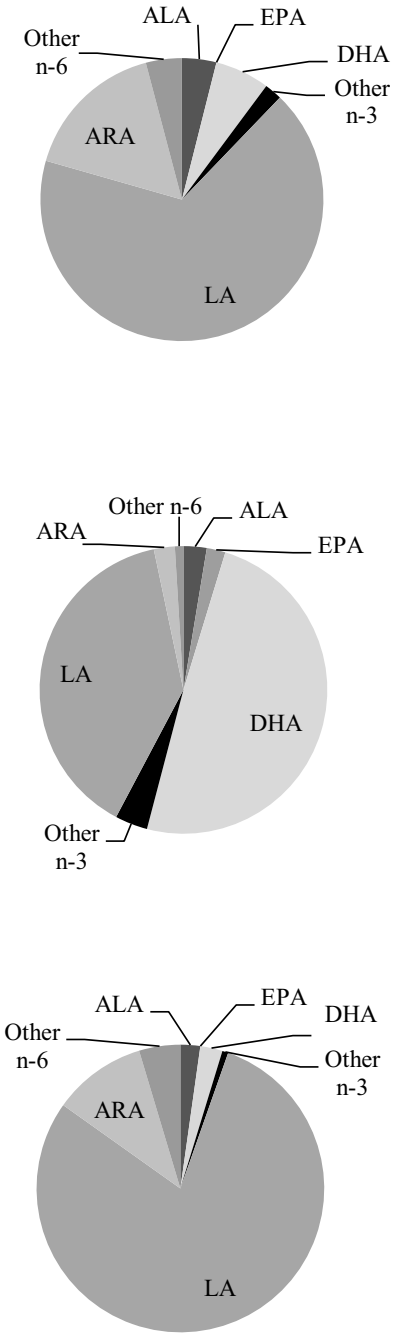

Fig. 1. Distribution of heart oxylipin and PUFA mass in phospholipid (PL) and neutral lipid (NL) fractions in rats provided the control (A), DHA (B) and linoleic acid (LA) (C) diets. Data shown are for combined data from female and male rats. Separate female and male data for all diet groups are provided in online Supplementary Table S4. ARA, arachidonic acid; ALA, $\alpha$-linolenic acid.

derived from different PUFA, so the effect of diet on the female: male ratio was examined separately for oxylipins grouped by their PUFA precursor. These analyses confirmed the generally higher levels of oxylipins in females (i.e. female:male ratios were predominantly greater than 1 ) and further demonstrated that the female:male ratio differed with different diets. Dietary ALA resulted in the highest female:male ratio $(>2)$ for all oxylipin groups, and dietary DHA was always among the lowest (0.51.5), with the remaining groups being in between (Fig. 3).

Sex effects on PUFA, however, were not consistent with sex effects on oxylipins. Main effects of sex were observed in three PUFA that were oxylipin precursors in the PL fraction: LA and ALA were higher in males, and DHA was higher in females. DGLA in the PL fraction also was higher in males, but only in rats given the control and ALA diets. In the NL fraction, the only oxylipin precursor with a sex effect was EPA, which was higher in females, but only in rats given the EPA diet. Details are provided in online Supplementary Tables S6 and S7.

\section{Oxylipin:PUFA ratios}

Oxylipin:PUFA ratios were calculated to compare the relative levels of oxylipins with their precursor PUFA in the PL fraction. In almost all cases, these ratios were higher in females compared with males, reflecting the higher oxylipin levels in females. With few exceptions, oxylipin:PUFA ratios were higher for $n-3$ compared with $n-6$ PUFA, and the order of oxylipin:PUFA ratios by chain length was 18 -carbon $\geq 20$-carbon $\geq 22$-carbon PUFA. A representative figure of these data is shown in Fig. 4 and data for all ratios can be found in online Supplementary Table S8.

\section{Discussion}

The data reported herein provide fundamental data on the rat heart oxylipin profile and demonstrate how it is affected by sex and by dietary oils enriched in ALA, EPA, DHA and LA. Compared with kidney and liver in these rats, the distribution 
(A)

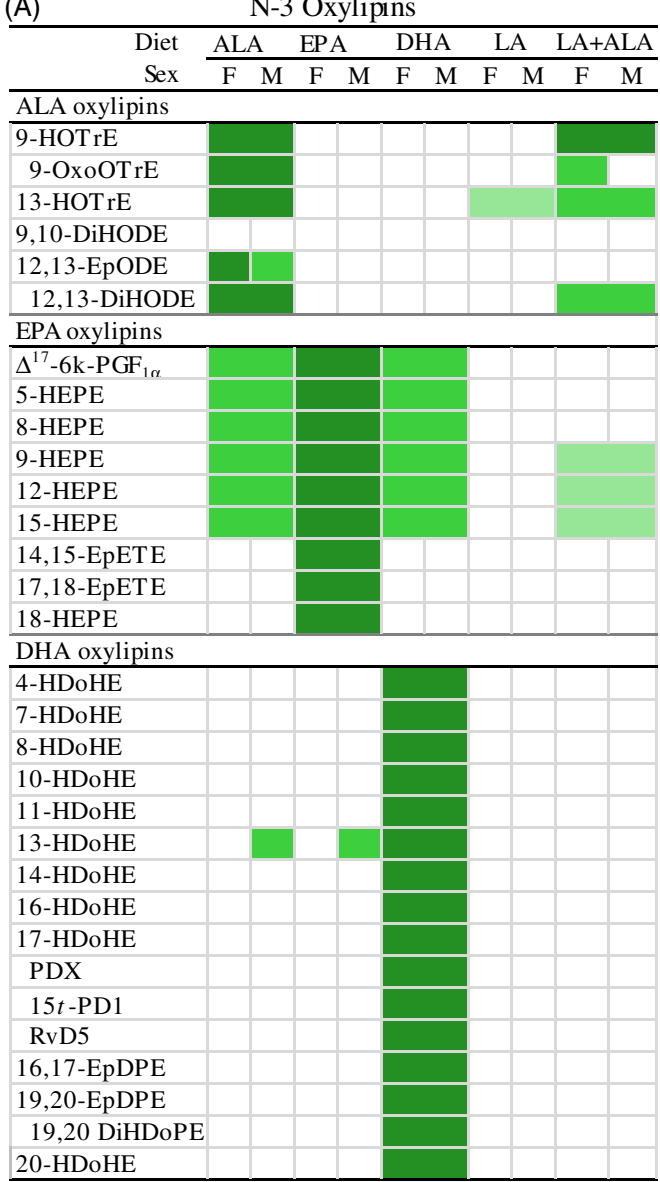

(C)

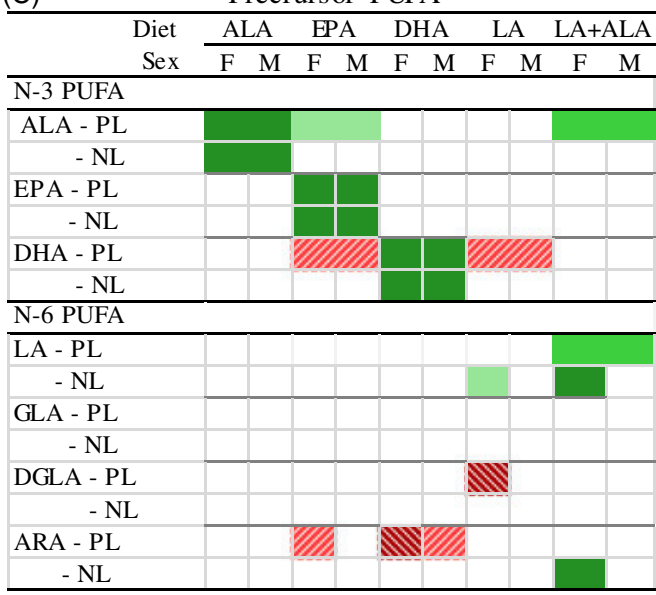

(B)

\begin{tabular}{|c|c|c|c|c|c|c|}
\hline Die & ALA & EPA & DHA & $\mathrm{LA}$ & & $\mathrm{A}+\mathrm{ALA}$ \\
\hline $\mathrm{Se}$ & $\mathrm{F} \quad \mathrm{M}$ & $\mathrm{F} \quad \mathrm{M}$ & $\mathrm{F} \quad \mathrm{M}$ & $\mathrm{F} \quad \mathrm{I}$ & & $\mathrm{M}$ \\
\hline
\end{tabular}

9-HODE

9-OxoODE

13-HODE

13-OxoODE

9,10,13-TriHOME

9,12,13-TriHOME

9,10-EpOME

9,10-DiHOME

12,13- EpOME

12,13-DiHOME

GLA oxylipins

13-HOTrE $\gamma$

DGLA Oxylipins

$\mathrm{PGF}_{1 \alpha}$

8-HETrE

15-HETrE

ARA oxylipins

$\mathrm{PGD}_{2}$

$15 \mathrm{~d}-\mathrm{PGD}_{2}$

$\mathrm{PGJ}_{2}$

$6 \mathrm{k}-\mathrm{PGF}_{1 \alpha}$

$\mathrm{PGE}_{2}$

$15 \mathrm{k}-\mathrm{PGE}_{2}$

$\mathrm{TxB}_{2}$

12-HHTrE

5-HETE

5-OxoETE

5,15-DiHETE

8-HETE

9-HETE

11-HETE

12-HETE

12-OxoETE

15-HETE

15-OxoETE

$6 t, 12$ epi-LTB ${ }_{4}$

$\mathrm{LTB}_{4}$

5,6-EpETrE

5,6-DiHETrE

8,9-EpETrE

8,9-DiHETrE

11,12-EpETrE

11,12-DiHETrE

14,15-EpETrE

14,15-DiHETrE

16-HETE

18-HETE

\section{Legend}

Shading of relative differences in PUFA and

oxylipins is as follows:

higher than control +1 other group
higher than control +2 or 3 other groups
higher than all other groups
lower than control +1 other group
lower than control +2 or 3 other groups
lower than all other groups

Fig. 2. (Colour online) Relative differences in heart (A) $n-3$ oxylipins, (B) $n-6$ oxylipins and (C) their precursor PUFA in rats provided ALA, EPA, DHA, linoleic acid (LA) and LA $+\alpha$-linolenic acid (ALA) diets compared with control diets. Data were analysed by two-way ANOVA when normally distributed and by Kruskal-Wallis when not, followed by Tukey's post hoc test. Cells are only shaded when there is a significant statistical difference $(P<0.05)$. Means, standard errors, $P$ values and complete statistical analysis of diet, sex and interaction effects are provided in online Supplementary Tables S2, S3, S6 and S7. ARA, arachidonic acid; d, deoxy; DGLA, dihomo- $\gamma$ linolenic acid; DiHDoPE, dihydroxy-docosapentaenoic acid; DiHETE, dihydroxy-eicosatetraenoic acid; DiHETrE, dihydroxy-eicosatrienoic acid; DiHODE, dihydroxyoctadecadienoic acid; DiHOME, dihydroxy-octadecenoic acid; EpDPE, epoxy-docosapentaenoic acid; EpETE, epoxy-eicosatetraenoic acid; EpETrE, epoxy-eicosatrienoic acid; EpODE, epoxy-octadecadienoic acid; EpOME, epoxy-octadecenoic acid; GLA, $\gamma$-linoleic acid; HDoHE, hydroxy-DHA; HEPE, hydroxy-eicosapentaenoic acid; HETE, hydroxy-eicosatetraenoic acid; HETrE, hydroxy-eicosatrienoic acid; HHTrE, hydroxy-heptadecatrienoic acid; HODE, hydroxy-octadecadienoic acid; HOTrE, hydroxy-octadecatrienoic acid; k, keto; LT, leukotriene; NL, neutral lipid; oxoETE, oxo-eicosatetraenoic acid; oxoODE, oxo-octadecadienoic acid; oxoOTrE, oxo-octadecatrienoic acid; PD, protectin; PL, phospholipid; Rv, resolvin; $t$, trans; TriHOME, trihydroxy-octadecenoic acid; Tx, thromboxane. 

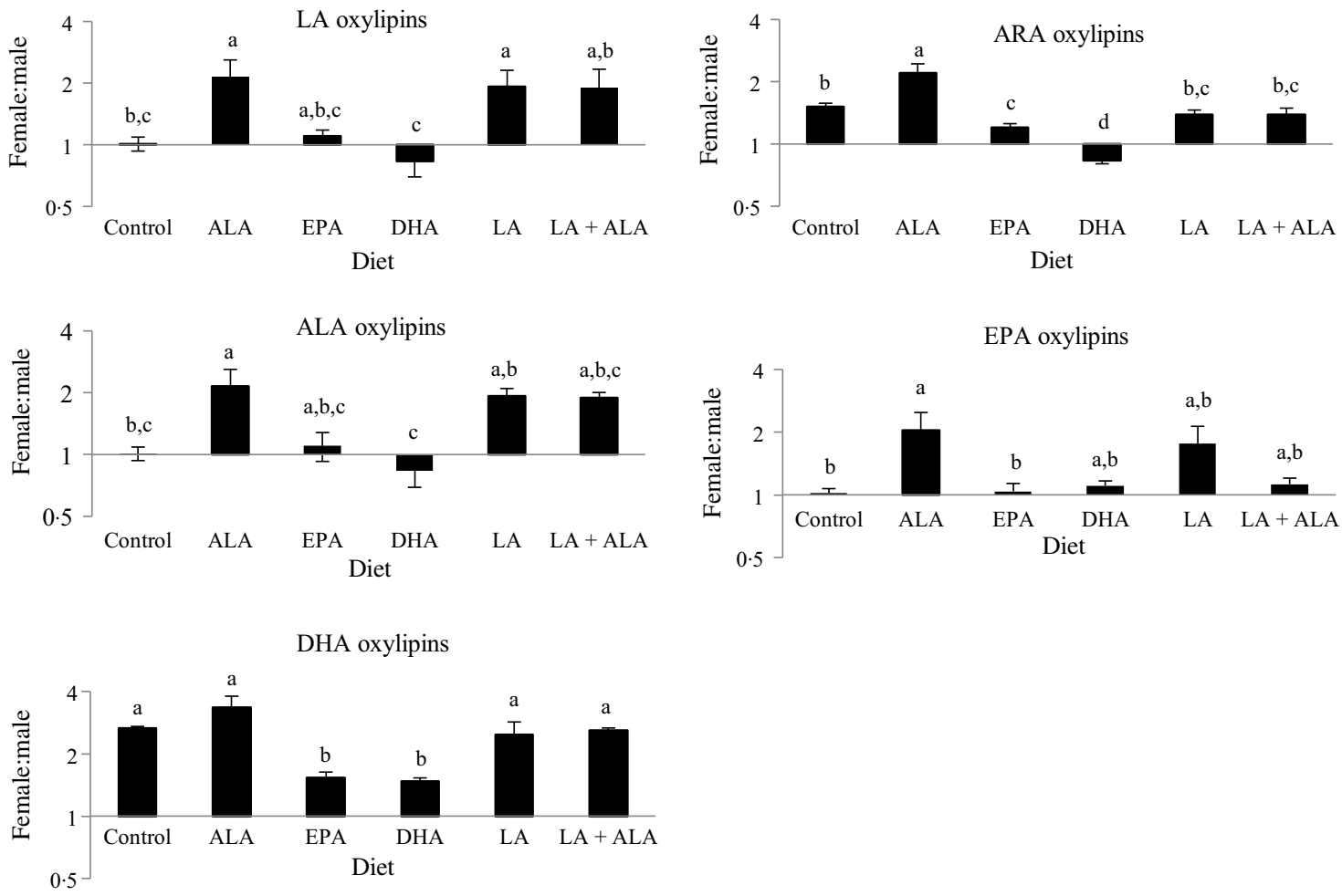

Fig. 3. Effect of diet on sex differences in oxylipin levels. Values are means, with their standard errors represented by vertical bars. Female oxylipin:male oxylipin mass ratios were analysed by one-way ANOVA when normally distributed and by Kruskal-Wallis when not, followed by Tukey's post hoc test. a,b,c,d Ratios with unlike letters are significantly different from each other $(P<0.05) . n 10$ for linoleic acid ((LA), 30 for arachidonic acid (ARA), 6 for $\alpha$-linolenic acid (ALA), 9 for EPA and 16 for DHA oxylipins.

of $n-6$ and $n-3$ PUFA-derived oxylipins was generally similar, with the exception of eleven detectable epoxygenated fatty acids (i.e. epoxy-octadecenoic acid, epoxy-octadecadienoic acid, EpETrE, epoxy-eicosatetraenoic acid, EpDPE) in the heart, compared with one in the kidney and three in the liver in these same rats ${ }^{(23)}$. These cytochrome-P450-derived oxylipins also have been reported in a study of mouse heart oxylipins ${ }^{(15)}$. Cytochrome P450 epoxygenase enzymes are highly expressed in heart ${ }^{(32,33)}$, and their epoxygenated products appear to be cardioprotective, especially in post-ischaemic states, with roles such as vasodilation via activation of $\mathrm{Ca}^{2+}$-sensitive $\mathrm{K}^{+}$channels, mitochondrial protection of cardiomyocytes, anti-apoptotic and pro-survival effects, and decreased cardiac fibrosis and inflammation effects ${ }^{(34-36)}$.

Altering the dietary oil content markedly changed the heart oxylipin profiles, as would be expected from the effects on their fatty acid precursors, and from what has been observed in other tissues in these rats ${ }^{(21,23,24)}$. The effects of individual $n-3$ PUFA could be evaluated because each diet was only enriched in one specific $n$-3 PUFA compared with the control diet. As in the other tissues in these rats, $n-3$ oxylipins were higher in rats provided the diets rich in their own individual PUFA precursor fatty acid. The heart compared with other tissues, however, was much more resistant to changes in oxylipins derived from other n-3 PUFA. EPA oxylipins were higher in hearts from rats provided the EPA, as well as the ALA and DHA diets, while ALA and DHA oxylipins were only elevated when rats were provided their direct precursor PUFA. In comparison, in kidney and some adipose depots, DHA oxylipins were higher in rats given diets enriched either in ALA, EPA or DHA. Heart oxylipins also changed less in response to dietary PUFA than the liver ${ }^{(24)}$, in which some ALA oxylipins were higher in rats given EPA and DHA diets, and in kidneys ${ }^{(23)}$ and some adipose depots, in which some ALA oxylipins were lower in rats given EPA and/or DHA diets $^{(21)}$. The apparent lack of effect of ALA or EPA on DHA oxylipins in the heart is supported by findings that the rat heart lacks elongase- $2^{(37)}$.

These findings may indicate that EPA oxylipins are key $n$-3-derived oxylipins in the heart, and a number of EPA oxylipins have beneficial effects in this regard. For example, 18-HEPE can prevent cardiac remodelling in the pressure-overload-induced mouse model ${ }^{(38)}$ and epoxygenated EPA such as 17,18-epoxy-eicosatetraenoic acid have antiarrhythmic effects in neonatal cardiomyocytes ${ }^{(39)}$ and vasodilatory effects in coronary smooth muscle cells ${ }^{(11)}$. Other EPA-derived eicosanoids such as $\mathrm{PGD}_{3}, \mathrm{PGE}_{3}$ and thromboxane $\mathrm{B}_{3}$ have lesser or no effects on inducing arrhythmias in cultured neonatal rat cardiac myocytes, when compared with their ARA-derived counterparts $^{(40)}$, and EPA-derived resolvin $\mathrm{E}_{1}$ can limit infarct size and ischemia-reperfusion injury in male rats ${ }^{(41)}$. Nevertheless, DHA oxylipins such as 13,14-EpDPE, 13,14-dihydroxy-DHA and 17,18-EpDPE have vasodilatory effects in coronary smooth muscle cells ${ }^{(11,42)}$ and 19,20-EpDPE has antiarrhythmic effects in neonatal cardiomyocytes ${ }^{(43)}$, indicating that these also have potential roles in the heart. To our knowledge, no effects of ALA oxylipins on heart function have been reported. 

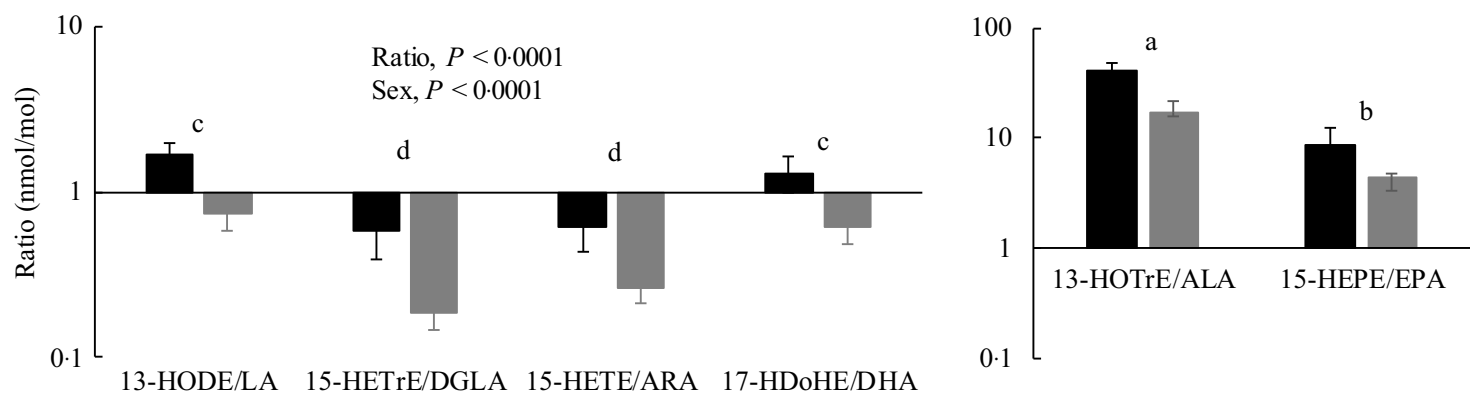

Fig. 4. Heart oxylipin:PUFA ratios for the 15 -lipoxygenase enzyme in rats ( $\square$, female; $\square$, male) provided the linoleic acid (LA) diet. Values are means, with their standard errors represented by vertical bars. Data were analysed by two-way ANOVA when normally distributed and by Kruskal-Wallis when not, followed by Tukey's post hoc test. ${ }^{a, b, b, c, d}$ Ratios with unlike letters are significantly different from each other $(P<0.05)$. All ratios for the $5-, 12-, 15$-lipoxygenase, cytochrome $P 450$ hydroxylase and epoxygenase enzymes for all diets are provided in online Supplementary Table S8. HODE, hydroxy-octadecadienoic acid; HETrE, hydroxy-eicosatrienoic acid; DGLA, dihomo- $\gamma$-linolenic acid; HETE, hydroxy-eicosatetraenoic acid; ARA, arachidonic acid; HDoHE, hydroxy-DHA; HOTrE, hydroxy-octadecatrienoic acid; ALA, $\alpha$-linolenic acid; HEPE, hydroxy-eicosapentaenoic acid.

In addition to these effects on $n$ - 3 oxylipins, dietary $n$ - 3 PUFA may also protect the heart by reducing the levels of $n$ - 6 oxylipins, particularly those derived from ARA. DHA was more potent in this regard, compared with both EPA and ALA, suggesting that DHA may have been more responsible for the reduction in the ARA oxylipins that has previously been observed in rat myocardium, as well as other tissues, with fish oil feeding ${ }^{(22,44,45)}$. ARA oxylipins are associated with several functional effects in the heart. For example, increased $\mathrm{PGF}_{2 \alpha}$ and $\mathrm{PGE}_{2}$ are associated with hypertrophic growth and production of arrhythmias in neonatal cardiac myocytes ${ }^{(40,46,47)}$, the lipoxygenase-generated HETE are increased in mitochondria from failing human hearts ${ }^{(48)}$ and 20-HETE induces vasoconstriction in small porcine coronary arteries ${ }^{(49)}$. Some effects of ARA oxylipins are protective, however, such as the vasodilatory EpETrE, which may prevent obesity-induced cardiomyopathy and reduce postischaemic ventricular polarisation in an isolated heart model ${ }^{(50,51)}$, and $\mathrm{PGI}_{2}$, which displays protective effects against the arrhythmias induced by other prostanoids in rat cardiac myocytes ${ }^{(40)}$. However, ARA oxylipins are more often associated with detrimental effects, while the EPA and DHA oxylipins are associated with cardio protective effects, as described above. Thus, the combination of higher levels of EPA (and DHA, and possibly ALA) oxylipins and lower levels of ARA oxylipins with ALA, EPA and DHA feeding would be predicted to have an overall protective effect on rat heart functions.

Increased dietary LA also has been promoted as heart healthy due to improvements in the blood lipoprotein profile, but the benefits of LA diets on CVD remain controversial ${ }^{(52-54)}$. Dietary LA increases renal and hepatic $n-6$ oxylipins derived from $n$-6 PUFA, including ARA ${ }^{(19,23)}$, suggesting that there may be effects on oxylipins that are not evident based on tissue fatty acid composition alone. In contrast to these tissues, dietary LA effects in the heart are restricted to increases in LA, $\gamma$-linolenic acid and DGLA oxylipins, further indicating greater resistance to changes in oxylipin composition by dietary oils in the heart compared with other tissues. Whether these oxylipins have significant effects on heart health, however, remains to be elucidated. On the one hand, the LA oxylipin 9,10-epoxy-octadecenoic acid can induce heart failure in $\operatorname{dogs}^{(55)}$, and its metabolite, 9,10-dihydroxy-octadecenoic acid, has been reported to decrease left ventricular-developed pressure recovery in the ischaemic/reperfused mouse heart ${ }^{(56)}$. On the other hand, the LA oxylipin, 13-hydroxy-octadecadienoic acid, and several DGLA oxylipins have been demonstrated to have anti-platelet effects ${ }^{(57-59)}$.

Interestingly, there also were several exceptions to these generalised patterns. For example, ARA oxylipins such as 15-deoxy-PGD 2 in EPA male, $\mathrm{PGJ}_{2}$ in DHA male and 15-keto$\mathrm{PGE}_{2}$ in (both male and female) EPA rats were increased while most of the other ARA oxylipins were decreased. Exceptions similar to this were observed in the kidney, liver and serum of these rats $^{(23)}$, as well as in human plasma after dietary intervention with individual EPA or docosapentaenoic acid $(22: 5 n-3)^{(60)}$. These PG metabolites are formed by hydroxy PG dehydrogenase (PGDH) activity ${ }^{(61)}$ and often have been considered to be inactive metabolites of their parent compounds. Although their functions are not well characterised, several studies report physiological effects. For example, 15-keto-PGE 2 can inhibit inflammatory cytokine production in acute liver injury ${ }^{(62)}$ and 15-PGDH activity suppresses colon tumorigenesis in vivo(63). How dietary $n$-3 PUFA affects PGDH activity or whether its oxylipins play functional roles in the heart by increasing these PG metabolites remains to be elucidated.

These data also illustrated other differences between fatty acid and oxylipin levels. For example, oxylipin product:PUFA precursor ratios are higher for shorter compared with longer chain PUFA and for $n-3$ compared with $n-6$ PUFA. This is similar to findings in other rat tissues ${ }^{(19,23,24)}$, and in studies of ARA compared with LA, and with EPA and DHA metabolism by 15-lipoxygenase ${ }^{(64,65)}$, and ARA compared with EPA and DHA metabolism by cytochrome P450 enzymes in vitro ${ }^{(39,66)}$. These findings may suggest that dietary 18 -carbon and $n$-3 PUFA may have greater effects on oxylipins in the heart. Thus, along with the greatest sex difference in oxylipins in ALA-fed rats, dietary ALA may have greater impact on heart physiology than suggested by fatty acid data alone.

The higher levels of DHA and lower levels of LA observed in female heart PL in the present study have previously been reported in the rat, although not always consistently ${ }^{(67,68)}$. Further we also observed higher ALA in male heart PL, which was not observed in these studies, possibly due to the inclusion 
of the high-ALA diet herein. However, to our knowledge, sex effects on the heart oxylipin profile have not been examined. In other tissues in these rats, we found that males generally have higher levels of oxylipins in kidney and liver ${ }^{(23)}$. In several adipose depots, oxylipin sex effects were observed in fewer oxylipins, with many having interaction effects. Interestingly, in those with interactions, they were higher in females given the control, ALA and EPA diets, but higher in males given the DHA diet ${ }^{(21)}$. This pattern is similar to the present findings in the heart where oxylipins in DHA rats were similar in females and males, but oxylipins in rats given the ALA diet were higher in females. The mechanism by which dietary PUFA interact with sex effects on heart oxylipins is not known, as this is a novel finding. In one study, a high-fat diet reduced renal cortical 20-HETE and EpETrE production in male but not female rats ${ }^{(69)}$, indicating that dietary fat may exert differential sex effects. However, the mechanisms by which sex alone, or in combination with diet, affects oxylipin levels remain largely unexplored in any tissue. There are examples of oestrogen increasing individual oxylipins and/or the enzymes that produce them ${ }^{(25,26,70)}$ or testosterone reducing these ${ }^{(71)}$. However, there are also studies which demonstrate higher levels of oxylipins in males or ovariectomised females ${ }^{(23,72,73)}$, so much remains to be elucidated in this regard. Interestingly, sex differences in oxylipins are exhibited to the greatest extent in oxylipins derived from DHA, compared with all other PUFA. This may be due to the higher levels of DHA in female heart PL, but this also remains to be investigated.

It should be noted that free oxylipins represent only a portion of the total oxylipins present in tissue. There is also a large pool of esterified oxylipins, but free oxylipins have been presumed to be the most active form and have been the most frequently studied. However, several reports also describe functions for esterified oxylipins ${ }^{(74,75)}$, and the presence of these in the heart would have an impact on the interpretation of the present data, although one study has reported that dietary modulation affects free and esterified oxylipins in a similar manner ${ }^{(76)}$.

\section{Conclusion}

In conclusion, this study provides novel data on the free oxylipin profile of the rat heart and on the differential effects of dietary $n-3$ and $n-6$ PUFA and sex on this profile. The dietary PUFA have the greatest effect on their own oxylipins, more so in the heart than other tissues. In addition, ARA oxylipins are markedly reduced by dietary $n$-3 PUFA with the effect being much greater with DHA compared with EPA and ALA. LA oxylipins, however, were largely unaffected by dietary $n$-3 PUFA, but were increased with higher dietary LA intake, even in the absence of increased LA in heart PL. Other discrepancies between oxylipin and fatty acid levels were observed for several PGDH-derived ARA oxylipin metabolites in EPA rats, for differences in oxylipin to parent PUFA ratios and for sex differences, further demonstrating that oxylipins do not necessarily reflect fatty acid compositions. Notably, these data show that sex differences in heart oxylipins interact with dietary PUFA effects, with dietary ALA compared with DHA resulting in higher levels of oxylipins in females. These fundamental data on heart oxylipins with unique dietary
PUFA and sex effects will help guide further investigations on the functions of oxylipins in the heart.

\section{Acknowledgements}

The authors thank Dennis Labossiere and Dennis Joseph for technical support and the St. Boniface Hospital Foundation for infrastructure support.

This study has been supported by the Natural Sciences and Engineering Research Council of Canada, the University of Manitoba Graduate Enhancement of Tri-Council Stipends (GETS) programme, Pizzey Ingredients and Manitoba Agriculture, Food and Rural Initiatives. Harold M. Aukema received grant support from Pizzey Ingredients.

A. F., S. L., H. M. A. designed the research; A. F., S. L., and T. W. conducted the research; A. F. and H. M. A. analysed the data, wrote the manuscript and had primary responsibility for final content.

The authors declare that there are no conflicts of interest.

\section{Supplementary material}

To view supplementary material for this article, please visit http://dx.doi.org/10.1017/S0007114519001211

\section{References}

1. Gabbs M, Leng S, Devassy JG, et al. (2015) Advances in our understanding of oxylipins derived from dietary PUFAs. Adv Nutr 6, 513-540.

2. Funk CD (2001) Prostaglandins and leukotrienes: advances in eicosanoid biology. Science 294, 1871-1875.

3. Li N, Liu JY, Qiu H, et al. (2011) Use of metabolomic profiling in the study of arachidonic acid metabolism in cardiovascular disease. Congest Heart Fail 17, 42-46.

4. Caligiuri SPB, Parikh M, Stamenkovic A, et al. (2017) Dietary modulation of oxylipins in cardiovascular disease and aging. Am J Physiol Heart Circ Physiol 313, H903-H918.

5. Gu X, Xu J, Zhu L, et al. (2016) Prostaglandin E2 reduces cardiac contractility via EP3 Receptor. Circ Heart Fail 9, e003291.

6. Elkhatali S, El-Sherbeni AA, Elshenawy OH, et al. (2015) 19-Hydroxyeicosatetraenoic acid and isoniazid protect against angiotensin II-induced cardiac hypertrophy. Toxicol Appl Pharmacol 289, 550-559.

7. Alsaad AM, Zordoky BN, Tse MM, et al. (2013) Role of cytochrome P450-mediated arachidonic acid metabolites in the pathogenesis of cardiac hypertrophy. Drug Metab Rev 45 , 173-195.

8. Dai M, Wu L, He Z, et al. (2015) Epoxyeicosatrienoic acids regulate macrophage polarization and prevent LPS-induced cardiac dysfunction. J Cell Physiol 230, 2108-2119.

9. Maayah ZH, El-Kadi AO (2016) 5-, 12- and 15-Hydroxyeicosatetraenoic acids induce cellular hypertrophy in the human ventricular cardiomyocyte, RL-14 cell line, through MAPK- and NF-kappaB-dependent mechanism. Arch Toxicol 90, 359-373.

10. Endo J, Sano M, Isobe Y, et al. (2014) 18-HEPE, an $n$-3 fatty acid metabolite released by macrophages, prevents pressure overload-induced maladaptive cardiac remodeling. I Exp Med 211, 1673-1687.

11. Ye D, Zhang D, Oltman C, et al. (2002) Cytochrome p- 450 epoxygenase metabolites of docosahexaenoate potently dilate 
coronary arterioles by activating large-conductance calciumactivated potassium channels. J Pharmacol Exp Ther 303, 768-776.

12. Hoffmann P, Mentz P, Blass KE, et al. (1982) Influence of dietary linoleic acid on cardiac function and prostaglandin release and on the effects of isoprenaline in the isolated rat heart. J Cardiovasc Pharmacol 4, 714-720.

13. Tloti MA, Moon DG, Weston LK, et al. (1991) Effect of 13-hydroxyoctadeca-9,11-dienoic acid (13-HODE) on thrombin induced platelet adherence to endothelial cells in vitro. Thromb Res 62, 305-317.

14. Viswanathan S, Hammock BD, Newman JW, et al. (2003) Involvement of CYP 2C9 in mediating the proinflammatory effects of linoleic acid in vascular endothelial cells. J Am Coll Nutr 22, 502-510.

15. Willenberg I, Rund K, Rong S, et al. (2016) Characterization of changes in plasma and tissue oxylipin levels in LPS and CLP induced murine sepsis. Inflamm Res 65, 133-142.

16. Pinto AM, Sanders TAB, Kendall AC, et al. (2017) A comparison of heart rate variability, $n$-3 PUFA status and lipid mediator profile in age-and BMI-matched middle-aged vegans and omnivores. Br J Nutr 117, 669-685.

17. Balvers MGJ, Verhoeckx KCM, Bijlsma S, et al. (2012) Fish oil and inflammatory status alter the $n-3$ to $n$ - 6 balance of the endocannabinoid and oxylipin metabolomes in mouse plasma and tissues. Metabolomics 8, 1130-1147.

18. Shearer GC, Harris WS, Pedersen TL, et al. (2010) Detection of omega-3 oxylipins in human plasma and response to treatment with omega-3 acid ethyl esters. J Lipid Res 51, 2074-2081.

19. Caligiuri SP, Love K, Winter T, et al. (2013) Dietary linoleic acid and $\alpha$-linolenic acid differentially affect renal oxylipins and phospholipid fatty acids in diet-induced obese rats. $J$ Nutr 143, 1421-1431.

20. Monirujjaman M, Devassy JG, Yamaguchi T, et al. (2017) Distinct oxylipin alterations in diverse models of cystic kidney diseases. Biochim Biophys Acta 1862, 1562-1574.

21. Mendonca AM, Cayer LGJ, Pauls SD, et al. (2018) Distinct effects of dietary ALA, EPA and DHA on rat adipose oxylipins vary by depot location and sex. Prostaglandins Leukot Essent Fatty Acids 129, 13-24.

22. Abeywardena MY \& Charnock JS (1995) Dietary lipid modification of myocardial eicosanoids following ischemia and reperfusion in the rat. Lipids 30, 1151-1156.

23. Leng S, Winter T, Aukema HM (2017) Dietary LA and sex effects on oxylipin profiles in rat kidney, liver, and serum differ from their effects on PUFAs. J Lipid Res 58, 1702-1712.

24. Leng S, Winter T \& Aukema HM (2018) Dietary ALA, EPA and DHA have distinct effects on oxylipin profiles in female and male rat kidney, liver and serum. J Nutr Biochem 57, 228-237.

25. Jun SS, Chen Z, Pace MC, et al. (1998) Estrogen upregulates cyclooxygenase-1 gene expression in ovine fetal pulmonary artery endothelium. J Clin Invest 102, 176-183.

26. Calkin AC, Sudhir K, Honisett S, et al. (2002) Rapid potentiation of endothelium-dependent vasodilation by estradiol in postmenopausal women is mediated via cyclooxygenase 2. J Clin Endocrinol Metab 87, 5072-5075.

27. Hall LM \& Murphy RC (1998) Electrospray mass spectrometric analysis of 5-hydroperoxy and 5-hydroxyeicosatetraenoic acids generated by lipid peroxidation of red blood cell ghost phospholipids. J Am Soc Mass Spectrom 9, 527-532.

28. Sankaran D, Lu J, Bankovic-Calic N, et al. (2004) Modulation of renal injury in pcy mice by dietary fat containing $n-3$ fatty acids depends on the level and type of fat. Lipids 39, 207-214.

29. Aukema HM, Yamaguchi T, Takahashi H, et al. (1992) Abnormal lipid and fatty acid compositions of kidneys from mice with polycystic kidney disease. Lipids 27, 429-435.
30. Aukema HM \& Holub BJ (1989) Effect of dietary supplementation with a fish oil concentrate on the alkenylacyl class of ethanolamine phospholipid in human platelets. J Lipid Res 30, 59-64.

31. Fair DE, Ogborn MR, Weiler HA, et al. (2004) Dietary soy protein attenuates renal disease progression after 1 and 3 weeks in Han:SPRD-cy weanling rats. J Nutr 134, 1504-1507.

32. Wu S, Chen W, Murphy E, et al. (1997) Molecular cloning, expression, and functional significance of a cytochrome P450 highly expressed in rat heart myocytes. J Biol Chem 272, 12551-12559.

33. Delozier TC, Kissling GE, Coulter SJ, et al. (2007) Detection of human CYP2C8, CYP2C9, and CYP2J2 in cardiovascular tissues. Drug Metab Dispos 35, 682-688.

34. Zhao G, Wang J, Xu X, et al. (2012) Epoxyeicosatrienoic acids protect rat hearts against tumor necrosis factor- $\alpha$-induced injury. J Lipid Res 53, 456-466.

35. Seubert JM, Sinal CJ, Graves J, et al. (2006) Role of soluble epoxide hydrolase in postischemic recovery of heart contractile function. Circ Res 99, 442-450.

36. Samokhvalov V, Alsaleh N, El-Sikhry HE, et al. (2013) Epoxyeicosatrienoic acids protect cardiac cells during starvation by modulating an autophagic response. Cell Death Dis $\mathbf{4}$, e885.

37. Igarashi M, Ma K, Chang L, et al. (2008) Rat heart cannot synthesize docosahexaenoic acid from circulating $\alpha$-linolenic acid because it lacks elongase-2. J Lipid Res 49, 1735-1745.

38. Endo J, Sano M, Isobe Y, et al. (2014) 18-HEPE, an $n$-3 fatty acid metabolite released by macrophages, prevents pressure overload-induced maladaptive cardiac remodeling. I Exp Med 211, 1673-1687.

39. Arnold C, Markovic M, Blossey K, et al. (2010) Arachidonic acid-metabolizing cytochrome $\mathrm{P} 450$ enzymes are targets of (47)-3 fatty acids. J Biol Chem 285, 32720-32733.

40. Li Y, Kang JX \& Leaf A (1997) Differential effects of various eicosanoids on the production or prevention of arrhythmias in cultured neonatal rat cardiac myocytes. Prostaglandins 54, 511-530

41. Keyes KT, Ye Y, Lin Y, et al. (2010) Resolvin E1 protects the rat heart against reperfusion injury. Am JPhysiol Heart Circ Physiol 299, H153-H164.

42. Li X, Hong S, Li PL, et al. (2011) Docosahexanoic acid-induced coronary arterial dilation: actions of 17S-hydroxy docosahexanoic acid on $\mathrm{K}+$ channel activity. J Pharmacol Exp Ther 336, 891-899.

43. Westphal C, Konkel A \& Schunck WH (2011) CYP-eicosanoidsa new link between omega-3 fatty acids and cardiac disease? Prostaglandins Other Lipid Mediat 96, 99-108.

44. Devassy JG, Yamaguchi T, Monirujjaman M, et al. (2017) Distinct effects of dietary flax compared to fish oil, soy protein compared to casein, and sex on the renal oxylipin profile in models of polycystic kidney disease. Prostaglandins Leukot Essent Fatty Acids 123, 1-13.

45. Ibrahim NH, Jia Y, Devassy JG, et al. (2014) Renal cyclooxygenase and lipoxygenase products are altered in polycystic kidneys and by dietary soy protein and fish oil treatment in the Han:SPRD-Cy rat. Mol Nutr Food Res 58, 768-781.

46. Adams JW, Migita DS, Yu MK, et al. (1996) Prostaglandin F2 $\alpha$ stimulates hypertrophic growth of cultured neonatal rat ventricular myocytes. J Biol Chem 271, 1179-1186.

47. Mendez M \& LaPointe MC (2005) PGE2-induced hypertrophy of cardiac myocytes involves EP4 receptor-dependent activation of p42/44 MAPK and EGFR transactivation. Am J Physiol Heart Circ Physiol 288, H2111-H2117.

48. Moon SH, Liu X, Cedars AM, et al. (2018) Heart failure-induced activation of phospholipase iPLA2 $\gamma$ generates hydroxyeicosatetraenoic acids opening the mitochondrial permeability transition pore. J Biol Chem 293, 115-129. 
49. Randriamboavonjy V, Busse R \& Fleming I (2003) 20-HETEinduced contraction of small coronary arteries depends on the activation of Rho-kinase. Hypertension 41, 801-806.

50. Cao J, Singh SP, McClung JA, et al. (2017) EET intervention on Wnt1, NOV, and HO-1 signaling prevents obesity-induced cardiomyopathy in obese mice. Am J Physiol Heart Circ Physiol 313, H368-H380.

51. Batchu SN, Law E, Brocks DR, et al. (2009) Epoxyeicosatrienoic acid prevents postischemic electrocardiogram abnormalities in an isolated heart model. J Mol Cell Cardiol 46, 67-74.

52. Harris WS, Mozaffarian D, Rimm E, et al. (2009) Omega-6 fatty acids and risk for cardiovascular disease. Circulation 119, 902-907.

53. Maki KC, Eren F, Cassens ME et al. (2018) $\omega-6$ Polyunsaturated fatty acids and cardiometabolic health: current evidence, controversies, and research gaps. Adv Nutr 9, 688-700.

54. Ramsden CE, Zamora D, Majchrzak-Hong S, et al. (2016) Re-evaluation of the traditional diet-heart hypothesis: analysis of recovered data from Minnesota Coronary Experiment (1968-73). BMJ 353, i1246.

55. Sugiyama S, Hayakawa M, Nagai S, et al. (1987) Leukotoxin, 9 , 10-epoxy-12-octadecenoate, causes cardiac failure in dogs. Life Sci $\mathbf{4 0}, 225-231$.

56. Edin ML, Wang Z, Bradbury JA, et al. (2011) Endothelial expression of human cytochrome P450 epoxygenase CYP2C8 increases susceptibility to ischemia-reperfusion injury in isolated mouse heart. FASEB J 25, 3436-3447.

57. Whitaker MO, Wyche A, Fitzpatrick F, et al. (1979) Triene prostaglandins: prostaglandin D3 and icosapentaenoic acid as potential antithrombotic substances. Proc Natl Acad Sci U S A 76, 5919-5923.

58. Westwick J (1976) The effect of pulmonary metabolites of prostaglandins E1, E2 and F2 $\alpha$ on ADP-induced aggregation of human and rabbit platelets [proceedings]. Br J Pharmacol 58, 297P-298P

59. Ikei KN, Yeung J, Apopa PL, et al. (2012) Investigations of human platelet-type 12-lipoxygenase: role of lipoxygenase products in platelet activation. J Lipid Res 53, 2546-2559.

60. Markworth JF, Kaur G, Miller EG, et al. (2016) Divergent shifts in lipid mediator profile following supplementation with $n-3$ docosapentaenoic acid and eicosapentaenoic acid. The FASEB J 30, 3714-3725.

61. Anggard E, Larsson C, Samuelsson B (1971) The distribution of 15-hydroxy prostaglandin dehydrogenase and prostaglandindelta 13-reductase in tissues of the swine. Acta Pbysiol Scand 81, 396-404.

62. Yao L, Chen W, Song K, et al. (2017) 15-hydroxyprostaglandin dehydrogenase (15-PGDH) prevents lipopolysaccharide (LPS)induced acute liver injury. PLOS ONE 12, e0176106.
63. Myung S-J, Rerko RM, Yan M, et al. (2006) 15Hydroxyprostaglandin dehydrogenase is an in vivo suppressor of colon tumorigenesis. Proc Natl Acad Sci U S A 103, 1209812102.

64. Soler J, Saura P, Garcia-Lopez D, et al. (2015) How can linoleic acid be the preferential substrate of the enzyme 15-lipoxygenase-1? A QM/MM approach. J Phys Chem B 120, 1950-1960.

65. Kutzner L, Goloshchapova K, Heydeck D, et al. (2017) Mammalian ALOX15 orthologs exhibit pronounced dual positional specificity with docosahexaenoic acid. Biochim Biophys Acta Mol Cell Biol Lipids 1862, 666-675.

66. Fischer R, Konkel A, Mehling H, et al (2014) Dietary omega-3 fatty acids modulate the eicosanoid profile in man primarily via the CYP-epoxygenase pathway. J Lipid Res 55, 1150-1164.

67. Slater-Jefferies JL, Hoile SP, Lillycrop KA et al. (2010) Effect of sex and dietary fat intake on the fatty acid composition of phospholipids and triacylglycerol in rat heart. Prostaglandins Leukot Essent Fatty Acids 83, 219-223.

68. Kitson AP, Smith TL, Marks KA et al. (2012) Tissue-specific sex differences in docosahexaenoic acid and $\Delta 6$-desaturase in rats fed a standard chow diet. Appl Physiol Nutr Metab 37, 1200-1211.

69. Zhou Y, Lin S, Chang H-H, et al. (2005) Gender differences of renal CYP-derived eicosanoid synthesis in rats fed a high-fat diet. American journal of hypertension 18, 530-537.

70. Geary GG, Krause DN, Duckles SP (2000) Estrogen reduces mouse cerebral artery tone through endothelial NOS- and cyclooxygenase-dependent mechanisms. Am J Physiol Heart Circ Physiol 279, H511-H519.

71. Pergola C, Rogge A, Dodt G, et al. (2011) Testosterone suppresses phospholipase $\mathrm{D}$, causing sex differences in leukotriene biosynthesis in human monocytes. FASEB J 25, 3377-3387.

72. Zuniga-Munoz AM, Guarner Lans V, Soria-Castro E, et al. (2015) $17 \beta$ estradiol modulates perfusion pressure and expression of 5-LOX and CYP450 4A in the isolated kidney of metabolic syndrome female rats. Int J Endocrinol, 149408.

73. Holla VR, Adas F, Imig JD, et al. (2001) Alterations in the regulation of androgen-sensitive Cyp 4A monooxygenases cause hypertension. Proc Natl Acad Sci U S A 98, 5211-5216.

74. O'Donnell VB, Murphy RC (2012) New families of bioactive oxidized phospholipids generated by immune cells: identification and signaling actions. Blood 120, 1985-1992.

75. Slatter DA, Percy CL, Allen-Redpath K, et al. (2018) Enzymatically oxidized phospholipids restore thrombin generation in coagulation factor deficiencies. JCI Insight 3, pii 98459.

76. Schebb NH, Ostermann AI, Yang J, et al. (2014) Comparison of the effects of long-chain omega-3 fatty acid supplementation on plasma levels of free and esterified oxylipins. Prostaglandins Other Lipid Mediat 113-115, 21-29. 